\title{
Diffusive Spreading of Chainlike Molecules on Surfaces
}

\author{
T. Ala-Nissila, ${ }^{1,2,3}$ S. Herminghaus, ${ }^{4,5}$ T. Hjelt, ${ }^{1,2}$ and P. Leiderer ${ }^{5}$ \\ ${ }^{1}$ Research Institute for Theoretical Physics, University of Helsinki, P.O. Box 9, FIN-00014 Helsinki, Finland \\ ${ }^{2}$ Laboratory of Physics, Tampere University of Technology, FIN-33101 Tampere, Finland \\ ${ }^{3}$ Department of Physics, Brown University, Providence, Rhode Island 02912 \\ ${ }^{4}$ Max-Planck Institute for Colloid and Interface Science, Rudower Chaussee 5, 12489 Berlin-Adlershof, Germany \\ ${ }^{5}$ Fakultät für Physik, Universität Konstanz, D-7750 Konstanz, Germany
}

(Received 30 January 1996)

\begin{abstract}
We study the diffusion and submonolayer spreading of chainlike molecules on surfaces. Using the fluctuating bond model, we extract the collective and tracer diffusion coefficients $D_{c}$ and $D_{t}$ with a variety of methods. We show that $D_{c}(\theta)$ has unusual behavior as a function of the coverage $\theta$. It first increases, but after a maximum goes to zero as $\theta \rightarrow 1$. We show that the increase is due to entropic repulsion that leads to steep density profiles for spreading droplets seen in experiments. We also develop an analytic model for $D_{c}(\theta)$ which agrees well with the simulations. [S0031-9007(96)00253-0]
\end{abstract}

PACS numbers: 68.35.Fx, 05.40.+j, 68.10.Gw

The spreading dynamics of molecularly thin oil films on solid substrates has gained substantial interest recently [1-5]. This is not only due to its obvious significance for technical applications, but also to the richness of the structures (layering, fingering) in the observed film profiles that sensitively depend on the molecular structure and interactions. As a typical feature, the development of molecularly thin so-called precursor films is observed [1]. The precursor film profile may be smooth or exhibit pronounced molecular layering effects ("dynamical layering").

At the foremost tip of the film, the flow dynamics develops into surface diffusion of single molecules, i.e., a $2 \mathrm{D}$ gas. In some experiments [2-4], it has been found that the transition from the dense submonolayer to the dilute film occurs much more rapidly than expected from simple molecular diffusion. The measured profiles are not Gaussian but assume a steeper shape that can be well fitted by a spherical cap [3] (see also Ref. [4]). An explanation for the late-time profiles has been suggested in terms of a transition of the fluid from a nonvolatile 3D to a volatile $2 \mathrm{D}$ phase where the attractive intermolecular interactions are weaker $[2,4]$. However, it is the aim of the present study to provide an alternative microscopic explanation for the steep density profiles observed in cases where desorption of the molecules can be neglected. By studying the diffusive properties of chainlike molecules, we demonstrate that such profiles can be generated by entropic repulsion alone.

To this end, we have performed a systematic study of diffusion and submonolayer spreading of athermal, flexible chains. We concentrate on the coverage dependence of collective and tracer diffusion coefficients $D_{c}(\theta)$ and $D_{t}(\theta)$, respectively. In addition to being important for submonolayer spreading dynamics, the diffusion of such molecules is of fundamental theoretical interest. Despite considerable experimental [6] and theoretical [7] work on adatom diffusion on surfaces there are only a few studies of more complicated molecules [8].

We use the fluctuating bond (FB) model with Monte Carlo (MC) simulations to extract both $D_{c}(\theta)$ and $D_{t}(\theta)$ from the relevant correlation functions in equilibrium. These results are complemented by analysis of simulated density profiles together with the nonlinear diffusion equation. We show that, while $D_{t}(\theta)$ is a decreasing function of $\theta$ as expected, $D_{c}(\theta)$ displays more complex behavior. It first increases with $\theta$, but after a maximum value goes to zero as $\theta \rightarrow 1$. We show that it is this positive slope of $D_{c}(\theta)$ that leads to steep precursor profiles in accordance with experiments [3]. We develop a thermodynamic theory for $D_{c}(\theta)$ which demonstrates that the initial increase is due to strong entropic repulsion, which is eventually overcome by the decreasing mobility of individual chains. The theory gives good agreement with our numerical results.

The FB model [9] is a 2D lattice model of polymer chains, where each segment excludes four nearest and next-nearest neighbor sites on a square lattice. The bond lengths $b_{\ell}$ are allowed to vary in the range $2 \leq b_{\ell} \leq \sqrt{13}$ (in units of the lattice constant), where the upper limit prevents bonds from crossing each other. The stiffness of the chains is controlled through an angle dependent potential $U_{\phi}=-\sum_{i=1}^{n} \sum_{j=1}^{N_{\mathrm{FB}}-1} \cos (\phi)$, where $n$ is the number of chains, $N_{\mathrm{FB}}$ is the number of segments in each chain, and $\phi$ is the angle between two adjacent bonds. Dynamics is introduced in the model by Metropolis moves of single segments, with a probability of acceptance $\min \left[e^{-\Delta U_{\phi} / T}, 1\right]$, where $\Delta U_{\phi}$ is the energy difference. Only such moves are allowed that obey both the site exclusion and the bond length restrictions. One MC time step is defined as one attempt to move each monomer of every chain. We note that, since no global translational modes of the chains are included, the rigid rod limit $T \rightarrow 0$ is not well defined in the model [10]. 
Simulations of diffusion were first done for $T=\infty$ using a $180 \times 180$ square lattice. We calculated $D_{t}$ from the definition

$$
D_{t}=\lim _{t \rightarrow \infty} \frac{1}{4 n t} \sum_{i=1}^{n}\left\langle\left|\vec{r}_{i}(t)-\vec{r}_{i}(0)\right|^{2}\right\rangle,
$$

where $\vec{r}_{i}(t)$ is the position of $i$ th particle at time $t$. The results for $N_{\mathrm{FB}}=6$ are shown in the inset of Fig. 1. As expected, $D_{t}(\theta)$ is a strongly decreasing function of $\theta$ due to the mutual blocking of the chains.

The collective diffusion coefficient, however, shows strikingly different behavior. It can be defined through the Green-Kubo relation [6] $D_{c}=\left[1 / 2\left\langle(\delta n)^{2}\right\rangle\right] \int_{0}^{\infty} d t\langle\vec{J}(0)$. $\vec{J}(t)\rangle$, where $\vec{J}(t)=\sum_{i=1}^{n} \vec{v}_{i}(t)$ is the total diffusion current and $\left\langle(\delta n)^{2}\right\rangle$ the mean square fluctuation (in a finite area $A$ ). Since the definition of $D_{c}$ involves cross-particle velocity-velocity correlations, it samples the average collective density fluctuations of the chains instead of just single-chain properties. In Fig. 1 we show results for $N_{\mathrm{FB}}=6$ conveniently obtained using the temporal decay of the Fourier transformed density autocorrelation function $S(k, t)=S_{0} e^{-k^{2} D_{C}(\theta) t}$ [11], where $S_{0}$ is a constant. Great care was taken to ensure that the hydrodynamic limit [6] was reached. Initially, $D_{c}(\theta)$ increases up to $\theta \approx 0.7$, after which it rapidly approaches zero.

The initial rise of $D_{c}(\theta)$ is in agreement with conclusions drawn from the experimental studies [12] and MC simulations of density profiles $[12,13]$. We did additional studies simulating a circular 2D droplet spreading using the FB model, and numerically solving the nonlinear diffusion equation $\partial \theta(x, t) / \partial t=\frac{\partial}{\partial x}\left[D_{c}(\theta) \partial \theta(x, t) / \partial x\right]$ by using a monotonically increasing tanh fitting function to $D_{c}(\theta)$ for $\theta<0.7$. By matching the simulated profiles to the solutions of the equation, the form of $D_{c}(\theta)$ can be determined. We found it to be in perfect agreement with

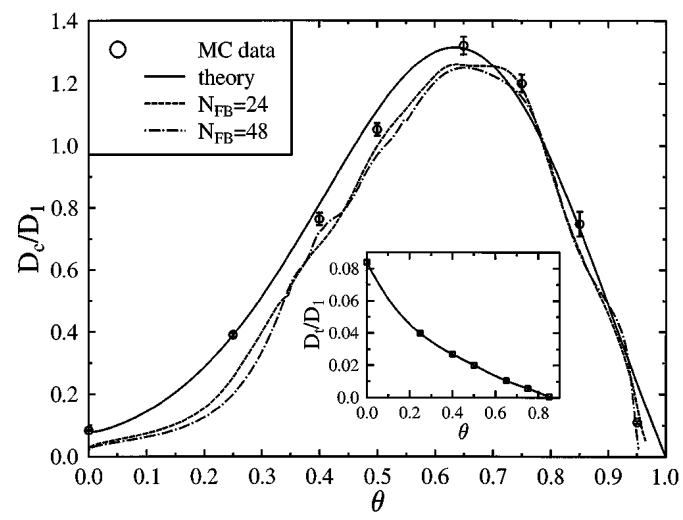

FIG. 1. Collective diffusion coefficient vs coverage for the FB model at $T=\infty$. The points are $\mathrm{MC}$ data for $N_{\mathrm{FB}}=6$, and the solid line is the theoretical fit with $N=16$. Dashed and dash-dotted lines are Boltzmann-Matano results for $N_{\mathrm{FB}}=24$ and 48. The inset shows the tracer diffusion coefficient for $N_{\mathrm{FB}}=6$. The curves have been normalized by $D_{1}$, which is the diffusion coefficient for a single segment. the MC results of Fig. 1. In Fig. 2 we show a comparison between experimental [3], simulated, and numerically calculated density profiles in the submonolayer regime. The agreement is excellent, and these profiles can be well fitted by a spherical cap shape with a Gaussian foot at lowest coverages [3].

Next, we studied the effects of chain length and stiffness to collective diffusion. To study the full coverage range, we performed careful Boltzmann-Matano analysis of spreading profiles $[6,14]$ in a rectangular geometry. The results for $N_{\mathrm{FB}}=6, T=\infty$ were checked against the $\mathrm{MC}$ data, and very good agreement was found. In Fig. 1 we show these data for $N_{\mathrm{FB}}=24$ and 48 , with $T=\infty$. As expected, diffusion slows down but the qualitative behavior remains the same. In Fig. 3 we show the effect of stiffness for $N_{\mathrm{FB}}=6$. Again, diffusion slows down but also now the maximum of $D_{c}(\theta)$ becomes less pronounced.

To explain the unusual behavior of $D_{c}(\theta)$, we consider a simple thermodynamic theory for collective diffusion [12]. The strong temperature dependence of $D_{c}(\theta)$ evident in Fig. 2 suggests that, besides the mobility of individual chains, entropy plays an important role here. Our starting point is the Nernst-Einstein equation [6], which relates collective diffusion to mobility $m$ via

$$
D_{c}(\theta)=m \theta\left(\frac{\partial \mu}{\partial \theta}\right)
$$

where $\mu$ is the chemical potential. For chainlike molecules, we approximate it by $\mu(\theta)=$ $\mu_{0}+k_{B} T[\ln (\rho)-\ln (w)]$, where $\rho=\theta / N$ is the number of molecules with $N$ segments per unit area and $w$ is the number of configurations of the chain. This

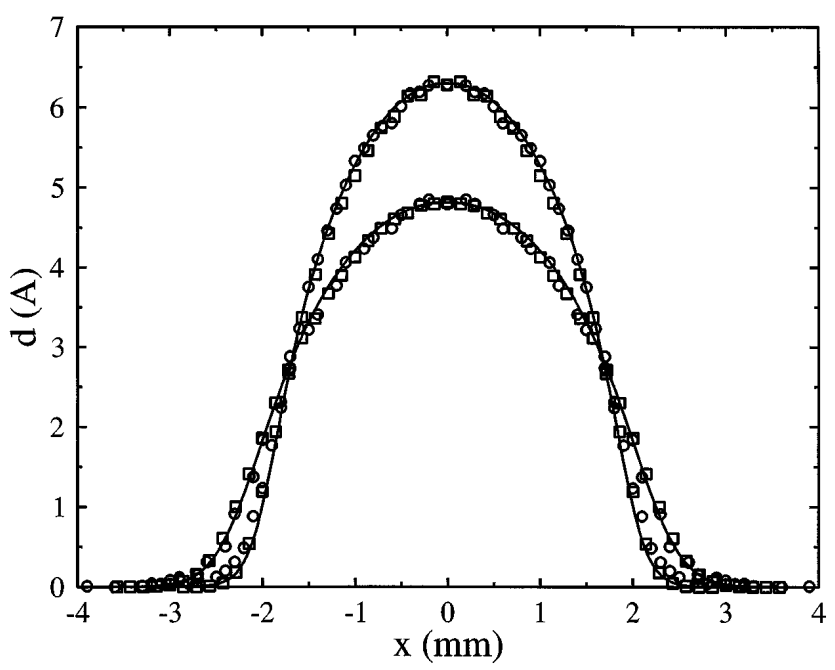

FIG. 2. Comparison between experimental density profiles of polydimethylsiloxine spreading on silver (circles), simulations of $2 \mathrm{D}$ circular droplets from the FB model with $N_{\mathrm{FB}}=6$ (squares), and numerical solutions of the 1D nonlinear diffusion equation (solid lines). 


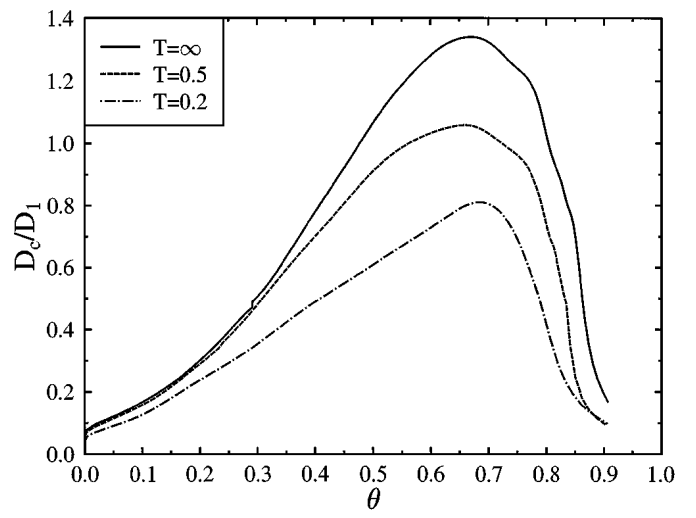

FIG. 3. The influence of chain stiffness for collective diffusion for $N_{\mathrm{FB}}=6$. The data have been obtained from Boltzmann-Matano analysis.

gives

$$
D_{c}(\theta)=m k_{B} T\left[1-\theta \frac{\partial \ln (w)}{\partial \theta}\right] .
$$

Next, both $m$ and $w$ must be estimated as a function of $\theta$. These quantities are model dependent. In general, the mobility is expected to decrease due to mutual blocking of the chains and may be approximated by $m \approx m_{0}[1-$ $\left.c_{n}(\theta)\right] \approx m_{0}\left(1-\theta^{\lambda}\right)$, where $m_{0}$ is a constant and $\lambda$ is a scaling exponent for the probability of nearest neighbor occupation $c_{n}$. Using Flory theory in $2 \mathrm{D}$, it can be argued that $\lambda \approx 2.5$ [12]. For the entropy, in the case of a lattice polymer with $N$ segments $(N \gg 1)$, we can similarly write $w \approx\left[1+q\left(1-c_{n}\right)\right]^{N}$, where $q>1$ is a parameter. With these approximations, $D_{c}$ becomes

$$
D_{c}(\theta)=D_{0}\left(1-\theta^{\lambda}\right)\left(1+\frac{\lambda N q \theta^{\lambda}}{1+q\left(1-\theta^{\lambda}\right)}\right)
$$

where $D_{0}$ is the single chain diffusion constant which for single segment dynamics scales $\propto 1 / N$. Thus, we can

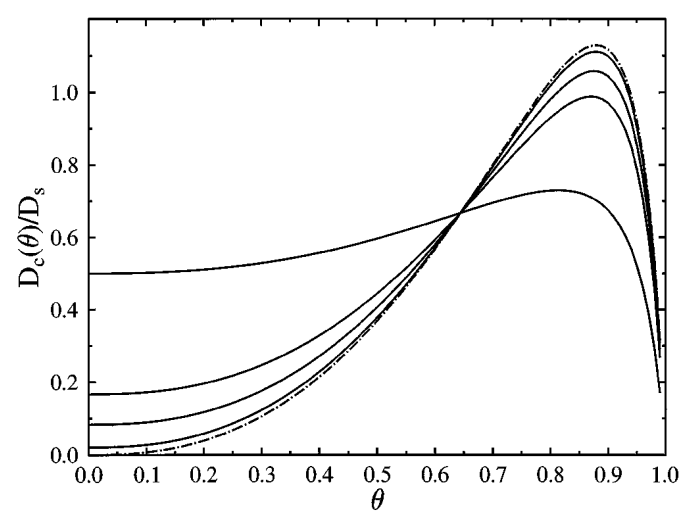

FIG. 4. $D_{c}(\theta) / D_{s}$ from Eq. (4), with $\lambda=2.5$ and $q=6$. The solid lines from top to bottom (at $\theta=0$ ) are for $N=$ $2,6,12$, and 48 . The maximum becomes more pronounced for large $N$, where Eq. (4) approaches a limiting form shown by the dashed line. write $D_{0}=D_{s} / N$. In Fig. 4 we show the behavior of this expression as a function of coverage for various values of $N$. The curves are strikingly similar to the results obtained for the FB model, and the maximum in $D_{c}(\theta)$ becomes relatively more pronounced for increasing $N$ [15].

The theory above reveals that the underlying reason for the unusual behavior of $D_{c}$ is entropic repulsion, which at higher coverages is overcome by decreasing mobility of single chains. For the FB model with $N_{\mathrm{FB}}=6$, we have numerically extracted an effective pair interaction potential $V_{e}(r)$ [16] which indeed shows a strong repulsion extending up to several lattice sites. We also find that all pair correlation functions $G(r)$ for coverages $\theta \leq 0.7$ and $N_{\mathrm{FB}}=6,24$, and 48 collapse to a single function which is given by $g(x)=G\left(r \theta^{\alpha} / N_{\mathrm{FB}}^{\beta}\right)$, where $\alpha \approx 0.38$ and $\beta \approx 0.55$. Finally, it is interesting to note that the maximum of $D_{c}(\theta)$ occurs at coverages close to typical critical fractional coverages for continuum percolation of 2D objects [17].

We can use the theory derived above to make a quantitative comparison with the FB model. To this end, we have calculated the mobility and find that $m \approx$ $D_{t}(0)\left(1-\theta^{1.4}\right)$ is a rather good approximation [18]. To estimate the entropy term for segments, we use the mean-field approximation $w=w_{1}^{2} w_{2}^{N-2}$, where $w_{1}$ is the entropy arising from a segment at the end of the chain and $w_{2}$ from one in the middle of the chain. For $N_{\mathrm{FB}}=6$, $T=\infty$ we have numerically determined $w_{1}(\theta)$ and $w_{2}(\theta)$, and find that they can be well fitted by a simple tanh function [19]. In Fig. 1 the solid line indicates the result for $D_{c}(\theta)$ as obtained from Eq. (3) where the only fitting parameter is $N$. We obtain very good agreement with $N=16$, which is reasonable since the true entropy is underestimated by our approximation.

To summarize, we have presented a combination of numerical and analytic arguments to explain how steep submonolayer density profiles observed in experiments [3] can be obtained solely from the entropic diffusion and spreading of chainlike molecules on surfaces. For athermal molecules, a strong entropic repulsion dominates at small and intermediate coverages, leading to the increase of $D_{c}(\theta)$. At high coverages, the chain mobility takes over and $D_{c}(\theta)$ approaches zero. The maximum of $D_{c}(\theta)$ is more pronounced for longer chains, but its position is relatively weakly dependent on the details of the model. This work also suggests that $D_{c}(\theta)$ can be used to obtain information about effective chain-chain interactions.

This work has been supported in part by the Academy of Finland. We wish to thank S.C. Ying for a critical reading of the manuscript.

[1] F. Heslot, N. Fraysse, and A. M. Cazabat, Nature (London) 338, 640 (1989); A. M. Cazabat, N. Fraysse, F. Heslot, P. Levinson, J. Marsh, F. Tiberg, and M. P. Valignat, Adv. Colloid Interface Sci. 48, 1 (1994). 
[2] A. M. Cazabat, N. Fraysse, F. Heslot, and P. Carles, J. Phys. Chem. 94, 7581 (1990).

[3] U. Albrecht, A. Otto, and P. Leiderer, Phys. Rev. Lett. 68, 3192 (1992); Surf. Sci. 283, 383 (1993).

[4] N. Fraysse, M. P. Valignat, A. M. Cazabat, F. Heslot, and P. Levinson, J. Colloid Interface Sci. 158, 27 (1993).

[5] M. Haataja, J. A. Nieminen, and T. Ala-Nissila, Phys. Rev. E 52, R2165 (1995), and references therein.

[6] R. Gomer, Rep. Prog. Phys. 53, 917 (1990).

[7] T. Ala-Nissila and S.C. Ying, Prog. Surf. Sci. 39, 227 (1992).

[8] M. V. Arena, E. D. Westre, and S. M. George, J. Chem. Phys. 94, 4001 (1991), and references therein.

[9] I. Carmesin and K. Kremer, Macromolecules 21, 2819 (1988).

[10] The present approximation for chain dynamics is valid in the limit where the time scale associated with activated jumps of single segments is much shorter than other microscopic time scales. It has been shown in Ref. [8] that for some molecules the diffusion barriers scale linearly with the chain length, which supports this approximation.
[11] C. H. Mak, H.C. Andersen, and S. M. George, J. Chem. Phys. 88, 4052 (1988).

[12] S. Herminghaus, U. Sigel, and P. Leiderer, Proceedings of the Moriond Meeting on Short and Long Chains at Interfaces, Villars-Sur-Ollon, 1995 (unpublished).

[13] T. Hjelt and T. Ala-Nissila (unpublished); S. Herminghaus, U. Sigel, U. Albrecht, and P. Leiderer (unpublished).

[14] C. Matano, Jpn. J. Phys. 8, 109 (1933).

[15] It should be noted that Eq. (4) does not give the correct result for pointlike particles, where $D_{c}=$ const. This limit can be built in by writing $\mu(\theta)=\mu_{0}+k_{B} T \ln [\theta /(1-$ $\theta)$ ] which is exact for a Langmuir gas [6], and $m \approx$ $D_{0}(1-\theta)$.

[16] L. Verlet, Phys. Rev. 165, 201 (1968).

[17] G. E. Pike and C. H. Seager, Phys. Rev. B 10, 1421 (1974).

[18] For the present system, this shows that setting $D_{t} \approx m$ [6] is a poor approximation, and correlation effects are important.

[19] For the FB model, fitting by $1+q\left(1-\theta^{\lambda}\right)$ gives unrealistic values for $q$ and $\lambda$, and the functional form is not well satisfied. 